\title{
Applicability and technical aspects of two-dimensional ultrasonography for assessment of fetal heart function
}

\author{
Nathalie Jeanne Magioli Bravo-Valenzuela ${ }^{1,2}$, Alberto Borges Peixoto ${ }^{2,3}$, Luciano Marcondes \\ Machado Nardozza ${ }^{2}$, Alex Sandro Rolland Souza ${ }^{4}$, Edward Araujo Júnior ${ }^{2}$
}

${ }^{1}$ Fetal echocardiography unit, University of Taubaté (UNITAU), Taubaté, state of São Paulo, ${ }^{2}$ Department of Obstetrics, Paulista School of Medicine, Federal University of São Paulo (EPM-UNIFESP), São Paulo, state of São Paulo, ${ }^{3}$ Mario Palmério University Hospital, University of Uberaba (UNIUBE), Uberaba, state of Minas Gerais, ${ }^{4}$ Department of Maternal and Child Health, Federal University of Pernambuco (UFPE), Recife, state of Pernambuco, Brazil

\section{Abstract}

In the recent years, fetal echocardiography has been used for the screening and diagnosis of anatomical heart defects and for the detailed study of fetal cardiac function. This method is characterized by its easy implementation and good reproducibility, allowing the diagnosis of myocardial dysfunction even in its subclinical phase. The functional assessment of the fetal heart should be routinely performed in fetuses with congenital heart disease and those without anatomical malformation. Several extra-cardiac conditions may alter fetal cardiac function, by increased placental resistance, volume overload or hyperdynamic circulation, compression, or maternal systemic disease with involvement of the fetal myocardium. This review addresses the main ultrasound techniques and various Doppler echocardiographic parameters available for the analysis of fetal heart function, and correlates them with clinical applicability. Various parameters available for the assessment of fetal myocardium, including those that evaluate atrial dynamics, can be used in this analysis and should be selected considering specific conditions.

Keywords: fetal heart function; 2D ultrasonography; color Doppler; M-mode; Tissue Doppler; myocardial performance index

\section{Introduction}

The prenatal screening for anatomical and functional congenital heart disease (CHD) by fetal ultrasound and echocardiography allows the early prenatal diagnosis and postnatal prognosis by allowing the planning of delivery, and in some cases, the administration of prenatal therapy [1]. A detailed assessment of cardiac function by

Received 19.12.2016 Accepted 20.01.2017

Med Ultrason

2017, Vol. 19, No 1, 94-101

Corresponding author: Prof. Edward ARAUJO JÚNIOR, $\mathrm{PhD}$

Rua Belchior de Azevedo, 156, apto. 111 -

Torre Vitoria, São Paulo, SP, Brazil

CEP 05089-030

Phone/Fax: +55-11-37965944

E-mail: araujojred@terra.com.br echocardiography is routinely performed in fetuses with CHD, and, recently, in those without anatomical heart defects, because it can assist in prenatal management and predict the perinatal and cardiovascular risks in the long term. Therefore, functional fetal echocardiography should be performed in fetuses without structural CHD who present extra-cardiac conditions, including fetal growth restriction (FGR), fetal tumors, twin-to-twin transfusion syndrome, fetal anemia, maternal congenital infections, and systemic diseases such as diabetes mellitus and chronic hypertension, which favor hemodynamic changes [2].

This review addresses the main techniques available for the assessment of fetal cardiac function and their clinical applications in conditions that may affect fetal hemodynamics and cardiovascular adaptation. 


\section{Fetal cardiovascular physiology}

To evaluate fetal cardiac function, it is important to understand the normal cardiac cycle, which involves systole and diastole. During ventricular systole, the heart contracts and ejects blood into the systemic circulation; and during diastole, there is myocardial relaxation, which allows blood entry into the ventricles [3].

The cardiac cycle can be subdivided into 5 phases (fig $1): 1)$ the isovolumetric relaxation phase, which is the time between the closing of the semilunar valves and the opening of the atrioventricular valves, without alterations in the intraventricular volume; 2) the fast filling phase, in which ventricular filling occurs, and the atrioventricular flow increases until it reaches a peak, corresponding to the $\mathrm{E}$ wave in the Doppler record of atrioventricular valve flow; 3 ) the active ventricular filling or atrial contraction phase, corresponding to the A wave in the Doppler record of the atrioventricular valves and in venous Doppler of the pulmonary vein, vena cava, and ductus venosus; 4) the isovolumetric contraction phase, which is the period between the closing of the mitral and tricuspid valves and the opening of the aortic and pulmonary valves; and 5) the ejection phase, in which the ventricular pressure increases enough to open the aortic and pulmonary valves and eject blood [3].

The fetal circulation has specific characteristics, including the communication between the left and right sides of the heart through the ductus venosus, foramen ovale, and ductus arteriosus [4]. Consequently, both ventricles are associated with systemic circulation; the right ventricle (RV) is responsible for irrigation of the lower half of the body and the left ventricle (LV) is responsible for irrigation of the brain, myocardium, and upper limbs. The aortic isthmus connects the blood flow from the upper to the lower parts of the fetal body. Because the ventricles are interdependent and the pulmonary venous blood returns through the inferior vena cava, the size of the foramen ovale and RV filling are important factors in the assessment of the function of LV. Similarly, LV function, vena cava flow, and size of the foramen ovale influence RV function. Therefore, the echocardiographic parameters used in the evaluation of postnatal cardiac function, while applicable to the fetal heart, are insufficient; other indices should therefore be added to evaluate atrial dynamics and the fetal venous system $[5,6]$.

Fetal heart failure occurs when the heart is unable to meet the metabolic needs of the tissues. The signs of fetal heart failure include cardiomegaly, atrioventricular valve insufficiency, and hydrops, which can be identified on ultrasonography [7]. The calculation of circumferential shortening fraction (delta-D) or ejection fraction using the

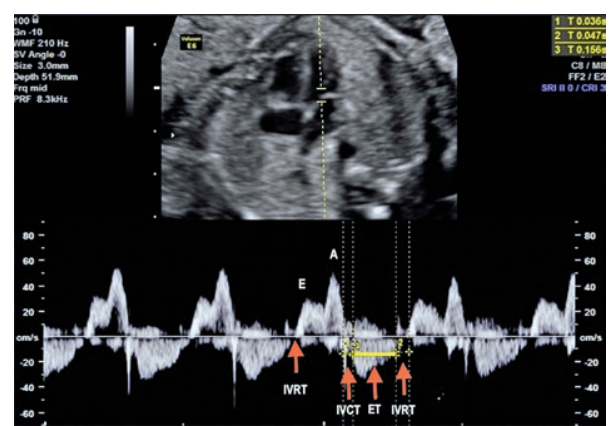

Fig 1. Doppler of the ventricular input and output flow, and the stages of the cardiac cycle: IVRT $=$ isovolumic relaxation time, rapid filling ( $\mathrm{E}$ wave), slow filling (A wave); IVCT = isovolumic contraction time; $\mathrm{ET}=$ ejection time.

M-mode allow for the assessment of global contractility of the fetal heart. However, this parameter only changes during the late phase of myocardial dysfunction. Considering that the heart undergoes remodeling at the precocious stages of heart dysfunction, the use of echocardiographic parameters is important for the early detection of changes in myocardial function. In most cases, diastolic changes precede systolic changes. Therefore, Doppler echocardiographic parameters that assess diastolic function or combined systolic and diastolic function, as well as the myocardial performance index (MPI), may contribute to the early treatment of fetuses with cardiac dysfunction [8].

In addition to MPI, several methods are used for the assessment of fetal cardiac function, such as cardiac biometry (cardiothoracic index [CTI]), cardiac cycle time, M-mode, two-dimensional color Doppler, conventional spectral Doppler, color tissue Doppler, and spectral tissue Doppler. Each of these methods has advantages and limitations [8].

\section{Cardiac biometrics}

The initial evaluation of a fetus with heart failure or risk of myocardial dysfunction includes the assessment of the presence or absence of cardiomegaly via a qualitative analysis and CTI. For calculation of CTI, it is necessary to identify the 4 chambers of the heart at a crosssectional plane of the thorax. CTI is the ratio between the cardiac and chest circumferences (normal values $\leq 0.5$ ) or the ratio between the cardiac and thoracic areas (normal values $\leq 0.35$ ) (fig 2) [6].

The increase of the cardiac area may be due to a primary disease of the myocardium, such as cardiomyopathy, or secondary to extra-cardiac causes, such as anemia, twin-to-twin transfusion, and infection. Generally, CTI is altered in conditions that involve global cardiomegaly. 


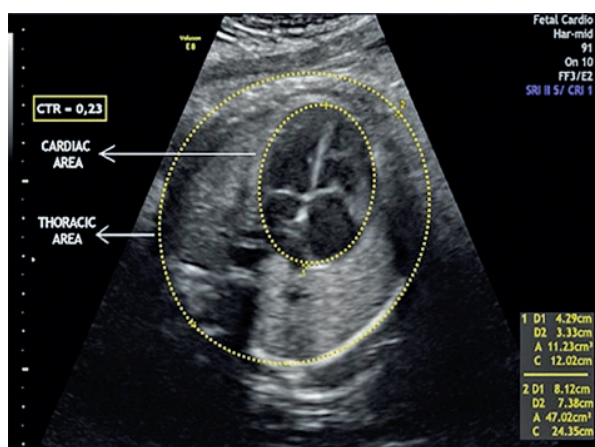

Fig 2. Relationship between the heart and chest areas obtained by planimetry in a transverse view of the fetal chest. CTI (cardiac area/thoracic area $=0.23$.

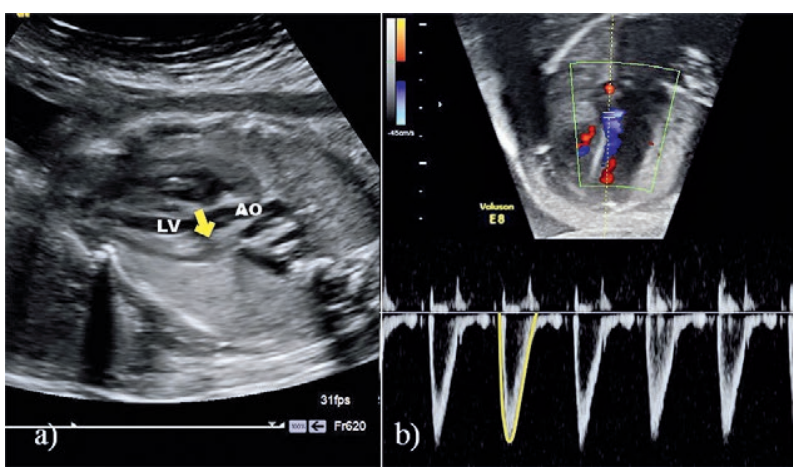

Fig 3. Calculation of the fetal stroke volume, or the volume ejected by the left ventricle, using the two-dimensional method: $\mathrm{VS}=$ diameter of the left ventricular outflow tract $\mathrm{x}$ VTI of the left ventricular outflow tract. a) Measurement of the left ventricular outflow tract in the apical five-chamber view (yellow arrow); b) VTI of the left ventricular outflow tract obtained in the five-chamber view. $\mathrm{SV}=$ stroke volume; $\mathrm{VTI}=$ mean velocity time integral. $\mathrm{LV}=$ left ventricle; Ao $=$ aorta.

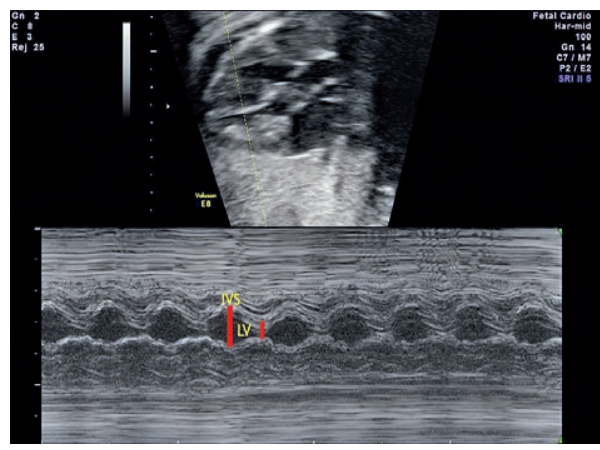

Fig 4. Calculation of shortening fraction (SF) or left ventricular delta using the M-mode at the longitudinal four-chamber view. $\mathrm{SF}=\mathrm{DDF}$ $-\mathrm{FSD} / \mathrm{DDF} . \mathrm{SF}=$ shortening fraction; $\mathrm{DDF}=$ maximum ventricular or tele-diastolic diameter; $\mathrm{DSF}=$ minimum ventricular or tele-systolic diameter; IVS = interventricular septum; LV = left ventricle.
Conversely, CTI is not altered in conditions that involve an increase in a single heart chamber, such as restrictive ductus arteriosus, in which an increase in the right chambers of the heart is observed.

Increased myocardial thickness can be easily identified and measured by two-dimensional echocardiography and M-mode. Myocardial hypertrophy is characterized by ventricular walls and interventricular septum $\geq 2$ standard deviations from the reference curve [9]. Owing to fetal hyperinsulinemia, maternal diabetes mellitus is the most common cause of hypertrophic cardiomyopathy [10]. In diabetes mellitus, myocardial hypertrophy is observed in the free ventricular wall, and most often in the interventricular septum. In FGR caused by placental insufficiency, LV hypertrophy and changes in cardiac geometry may occur, characterized by more a globular heart. This modification can be assessed using the LV sphericity index (the ratio between the longitudinal and transverse diameters of $\mathrm{LV}=0.5$ ) [11].

\section{Cardiac output (CO)}

Stroke volume (SV), or ejected volume, is calculated for each ventricle using the two-dimensional (2D) method, by multiplying the $\mathrm{CO}$ valve area by the mean velocity time integral (VTI) of the ventricular outflow tract (fig 3). It can also be calculated using the three-dimensional method (3D) and the following formula: end-diastolic volume (EDV) - end-systolic volume. The combined CO can be calculated by multiplying the sum of VS of both ventricles by the heart rate (bpm) $(\mathrm{CO}=\mathrm{VS}-\mathrm{VD}+\mathrm{VS}-\mathrm{VE} \mathrm{x}$ heart rate). As occurs with $\mathrm{SV}, \mathrm{CO}$ increases with gestational age; values from 400 to $500 \mathrm{ml} / \mathrm{min} / \mathrm{kg}$ are considered normal [12]. CO increases in situations such as arteriovenous fistulae, teratomas, and twin-to-twin transfusion, and decreases in cases of deficit of global contractility.

\section{Ejection fraction and circumferential shortening fraction or delta-D}

The maximum and minimum diameters of each ventricle can be obtained using the one-dimensional mode (M-mode) in the longitudinal four-chamber view or in the transventricular plane; these diameters are measured at end-diastole (EDD) and end-systole (ESD), respectively. This index can be calculated separately for each ventricle using the following formula: EDD - ESD/EDD (fig 4) and corresponds to the systolic function; because it assesses the global radial/circumferential contractility, it presents alterations in a later phase of systolic dysfunction. Circumferential shortening fraction (cSF) values smaller than 0.28 are considered altered $[7,13]$. 


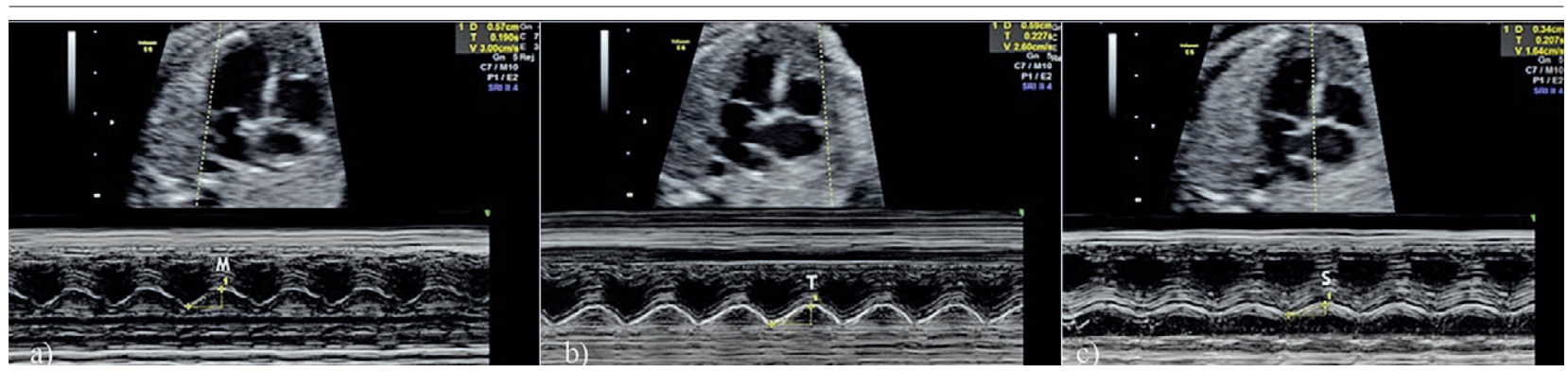

Fig 5. In the apical four-chamber view of the fetal heart, the M-mode image is positioned at the junction between the free ventricular wall and the atrioventricular valve, or between the ventricular wall and interventricular septum, to measure the maximum systolic excursion of the mitral valve, tricuspid valve, or interventricular septum, for the calculation of MAPSE (a), TAPSE (b), and SAPSE (c), respectively. $\mathrm{M}=\operatorname{MAPSE}(5.7 \mathrm{~mm}) ; \mathrm{T}=\operatorname{TAPSE}(5.9 \mathrm{~mm}) ; \mathrm{S}=\operatorname{SAPSE}(3.4 \mathrm{~mm})$.

\section{Fetal mitral, tricuspid, and interventricular septum annular plane systolic excursions (MAPSE, TAPSE, and SAPSE)}

These parameters allow the assessment of the longitudinal systolic function by measuring the atrioventricular annular movement using one-dimensional mode (M-mode) echocardiography. For this purpose, the apical four-chamber view needs to be obtained. The maximum systolic excursion of the respective valves is measured by positioning the M-mode image at the junction between the mitral annulus (MAPSE), tricuspid annulus (TAPSE), and free ventricular wall. The maximum systolic excursion of the interventricular septum (SAPSE) is measured by positioning the M-mode on the crux of the heart (fig 5). This parameter is easy to obtain and correlates well with tissue Doppler for the assessment of the diastolic function of the fetal myocardium [14]. The TAPSE and MAPSE reference values increase with gestational age; however, TAPSE values are higher than those of MAPSE $[15,16]$.

\section{Derivative of pressure with respect to time (dP/dt)}

In the presence of tricuspid or mitral regurgitation, the systolic performance of RV or LV can be quantified by dividing the change between the RV-RA or LV-LA gradient to the other $(\mathrm{dP})$ by the time interval for this variation (dt) (fig 6). $\mathrm{dP} / \mathrm{dt}$ values greater than $800 \mathrm{mmHg} / \mathrm{s}$ are considered normal whereas values smaller than 400 $\mathrm{mmHg} / \mathrm{s}$ indicate severe contractile deficit [17].

\section{Myocardial performance index (MPI)}

MPI, or Tei index, is a quantitative, non-invasive method used for the assessment of systolic and diastolic function [18]. MPI is obtained by recording the flow of the mitral and tricuspid valves, either by conventional or tissue Doppler; it also allows for the measurement of

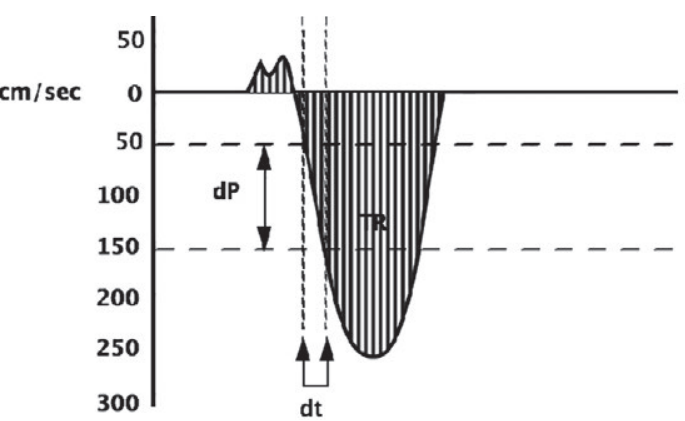

Fig 6. Derivative of pressure with respect to time (dP/dt) of tricuspid insufficiency, calculated by dividing the change in pressure $(\mathrm{dP})$ by the time interval $(\mathrm{dt})$ for this variation.

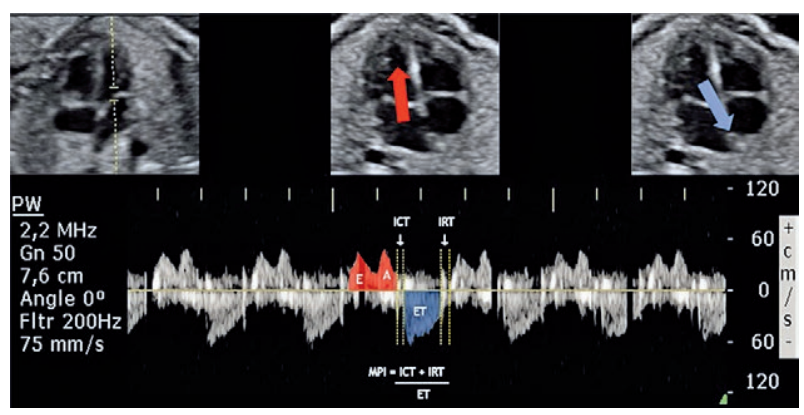

Fig 7. Myocardial performance index (MPI) of the left ventricle, calculated by pulsed Doppler with the input and output from the left ventricle (LV). Isovolumetric contraction time (IVCT) was determined from the beginning of the mitral valve closure to the opening of the aortic valve; ejection time (ET) was determined from the beginning of the opening to the beginning of the closure of the aortic valve; isovolumetric relaxation time (IVRT) was determined from the beginning of the aortic valve closure to the beginning of the opening of the mitral valve. MPI = IVCT + IVRT/ET.

each interval of the cardiac cycle. MPI can be calculated using the formula: isovolumic contraction time (IVCT) + isovolumic relaxation time (IVRT)/ejection time (ET) (fig 7). In practice, the sum of IVCT and IVRT can be calculated by the difference in the interval between the 
closing and opening of the atrioventricular valve (interval a) and ET (interval b). Myocardial dysfunction may increase the isovolumic intervals and decrease ET, which increases MPI. MPI values remain relatively stable and present a very slight variation during pregnancy [19].

IVCT corresponds to the time between ventricular contraction and the opening of the aortic and pulmonary valves. During this period, the intraventricular pressure increases, but the RV and LV volumes do not change. IVRT starts with diastole, after the closure of the aortic and pulmonary valves. At this time, ventricular isovolumetric relaxation occurs, blood inflow/outflow to the ventricles does not change, and the intraventricular pressure declines gradually. ET starts when the pressure within the ventricles is sufficient to promote the opening of the aortic and pulmonary valves; myocardial contraction takes place, and the blood reaches the systemic circulation [18].

MPI has a high sensitivity and specificity for predicting FGR, feto-fetal transfusion syndrome (receiver fetus), and perinatal morbidity and mortality in fetuses of pregnant women with diabetes mellitus [19].

\section{E/A ratio}

The classic assessment of fetal diastolic function uses the mitral and tricuspid valve flows to calculate the relationship between the $\mathrm{E}$ wave (passive ventricular filling) and A wave (atrial contraction) (fig 8). After the ninth week of pregnancy, the E and A waves can be measured; the E/A ratio is always lower than 1.0 until term, as the fetal heart is more rigid owing to its higher collagen content. For accurate measurement of $\mathrm{E}$ and $\mathrm{A}$ waves, either mitral or tricuspid, the sample volume of pulsed Doppler $(2-3 \mathrm{~mm})$ should be positioned in each ventricle immediately distal to the atrioventricular valves at an insonation angle smaller than $20^{\circ}$ [8].

The E/A ratio can be obtained using tissue Doppler and spectral Doppler. Tissue Doppler is more sensitive than spectral Doppler, does not change with gestational age, and allows for the evaluation of segmental diastolic

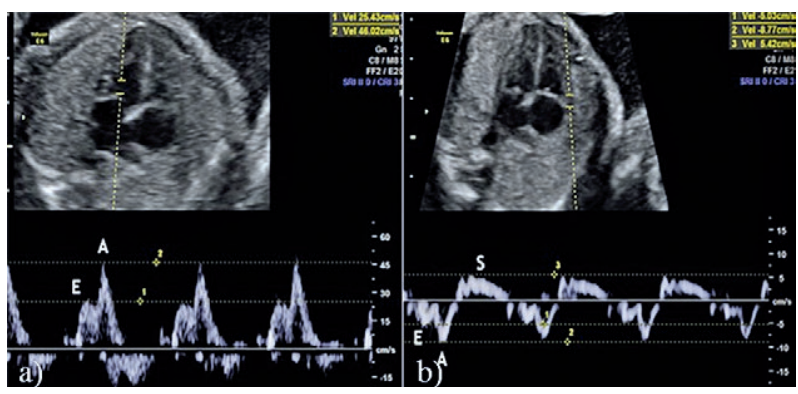

Fig 8. A/E ratio assessed by pulsed Doppler of the mitral valve (a) and lateral tissue Doppler of the right ventricle (b). function in the mitral and tricuspid annular (medial tissue), septal, and ventricular wall (lateral tissue) planes [20].

\section{Excursion index of the septum primum}

Considering that the ventricles are interdependent and communicate through the foramen ovale, changes in LV compliance and relaxation can reduce the movement of the foramen ovale membrane (septum primum), as noted in fetuses of pregnant women with diabetes mellitus and septal hypertrophy, and in cases of FGR [20,21]. This movement is quantified using the excursion index of the septum primum; this index is calculated by the ratio between the maximum diameter of the linear displacement of the septum primum at end-diastole and the maximum left atrial diameter at the four-chamber view (fig 9) [22]. This index is correlated with atrial dynamics, is used to evaluate diastolic function, and reflects the LV compliance.

\section{Left atrial shortening fraction}

The shortening fraction of the left atrium (LA) depends on the preload and is proportional to the ventricular compliance; it is calculated by dividing the result of the difference between the end-diastole and pre-systolic LA

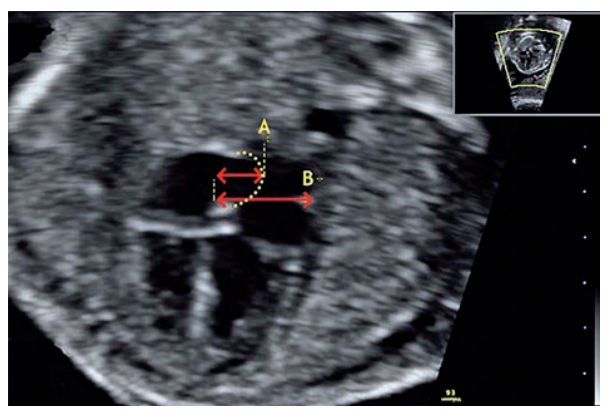

Fig 9. Septum primum excursion index: ratio of the maximum displacement of the septum primum (A) and the maximum diameter of the left atrium in a four-chamber view. Excursion index of the septum primum $(\mathrm{EISP})=\mathrm{A} / \mathrm{B}$.

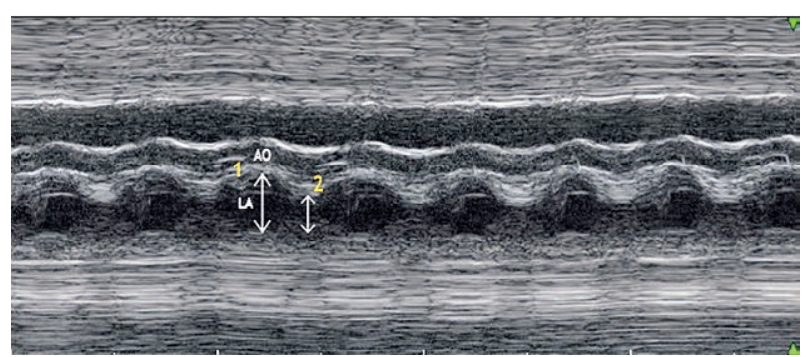

Fig 10. Left atrial shortening fraction: ratio between the maximum (1) and minimum diameters (2) of the left atrium (LA) along an axial plane of LV. LA = left atrium; Ao $=$ aorta. 
measurements by the end-diastole LA diameter on Mmode (normal values $>0.45$ ) (fig 10 ). AE cSF is correlated with atrial dynamics and reflects the LV compliance [22].

\section{Pulsatility index of the foramen ovale and pulmonary vein}

The pulsatility index (PI) of the foramen ovale and pulmonary vein can be calculated using the following formula: peak maximum velocity - peak pre-systolic velocity/average velocity. The blood flow through the foramen ovale should be analyzed by positioning the sample volume of the pulsed Doppler on the left atrial surface of the foramen ovale orifice at an angle smaller than $20^{\circ}$. The pulmonary vein PI can be obtained by positioning the sample volume of the pulsed Doppler at the right superior pulmonary vein, as close to its junction with $\mathrm{AE}$ as possible [23]. In situations of increased left atrial pressure, such as in fetuses of pregnant women with diabetes mellitus, myocardial hypertrophy, and FGR, pre-systolic velocity is reduced, with increased foramen ovale PI $(>2.5)$ and pulmonary vein PI (>1.2) (fig 11) [24].

\section{Pulsatily index of ductus venosus}

The ductus venosus (DV) has an important role in fetal hemodynamics, as the saturated blood flow through the foramen ovale depends on the flow rate coming from DV. Under physiological conditions, DV is triphasic and presents a positive A wave (fig 12). It reflects the right atrial dynamics and the preload dynamic of RV. In various pathological conditions with fetal deterioration, such as in FGR, PI decreases and the A wave decreases or reverses $[25,26]$.

\section{Isthmus flow index}

The aortic isthmus flow is functionally the only true arterial shunt in the fetus; it depends on the volume ejected by the 2 ventricles and the resistance of the fetal brain and placenta. The isthmus flow index (IFI) is obtained by positioning the sample volume of pulse Doppler in the region of the aortic isthmus, near the left subclavian artery in the longitudinal plane, and is calculated using the formula: systolic VTI+diastolic VTI/systolic VTI (normal IFI >1.2) (fig 13) [27,28].

\section{Scores of fetal myocardial function}

A cardiovascular score developed for the assessment of fetal heart failure in fetuses at risk of hydrops was validated by Hofstaetter et al [29] and can be used as a pre-

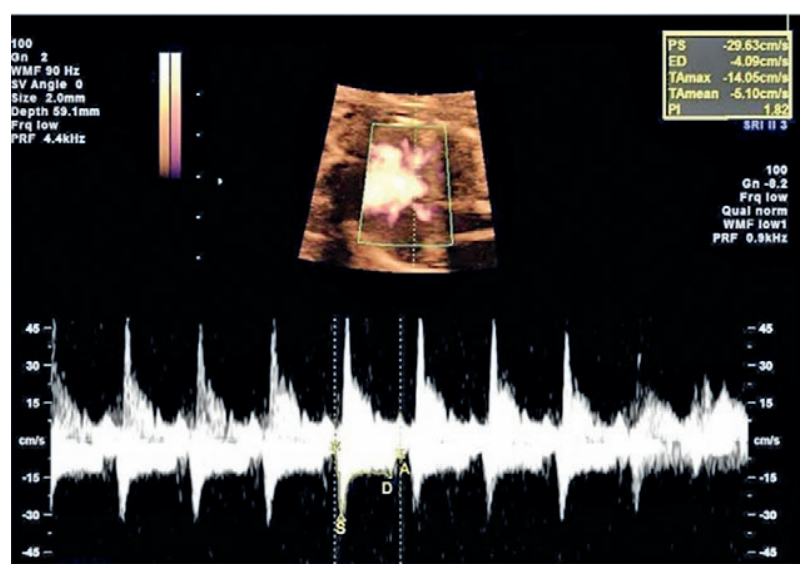

Fig 11. Pulmonary vein Doppler obtained in a four-chamber view at the venoatrial junction, right superior pulmonary vein, and the left atrium, showing a triphasic flow (S, D, and A waves) and increased pulsatility index (PI; 1.82) in a fetus with intrauterine growth restriction. $\mathrm{PI}=$ maximum velocity $(\mathrm{S}$ or D wave) - pre-systolic velocity (wave A)/wave (normal values $\leq 1.2$ ).

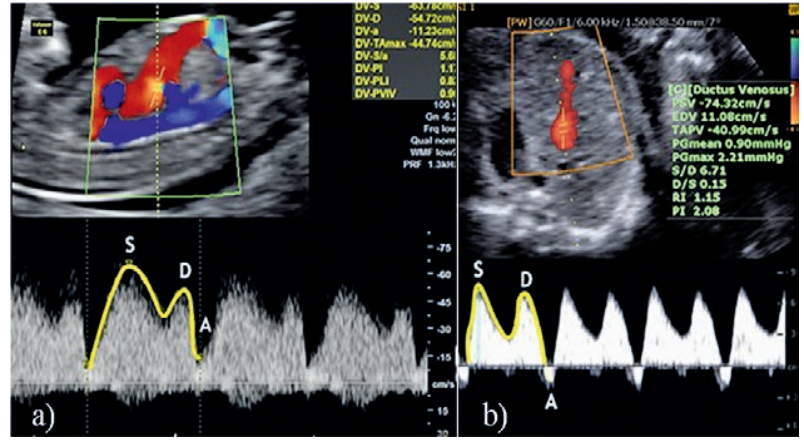

Fig 12. Doppler of the ductus venosus with normal triphasic flow (a) obtained with the sample volume of the pulsed Doppler in the sagittal plane and reverse A wave (b). S = systolic wave; $\mathrm{D}=$ diastolic wave; $\mathrm{A}=$ pre-systolic wave.

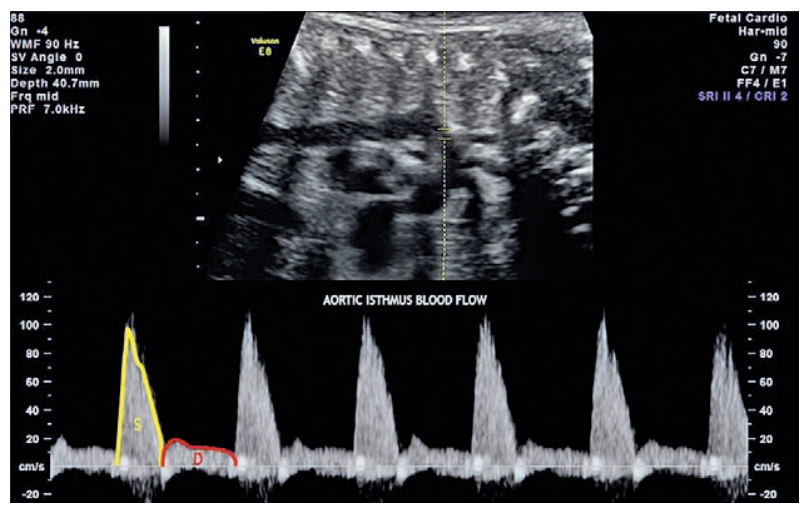

Fig 13. Isthmus flow index (IFI) with the placement of the sample volume of pulsed Doppler in the aortic isthmus, near the left subclavian artery in the longitudinal plane of the aortic arch. $\mathrm{IFI}=$ systole velocity time integral (VTI) - diastole VTI/systole VTI. $\mathrm{S}=$ systole; $\mathrm{D}=$ diastole. 


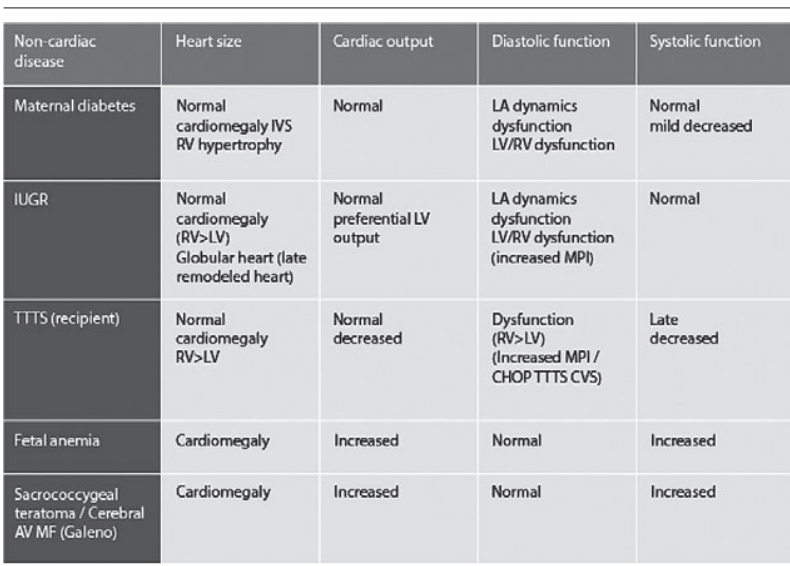

Fig 14. Parameters used in the cardiovascular score for fetal conditions involving risk of heart failure.

dictor of risk of perinatal morbidity and mortality. This score includes some of the parameters described above, such as fractional shortening, Doppler of DV, and cardiac biometry (CTI). A score of 10 is considered normal and values smaller than 7 indicate increased perinatal morbidity and mortality (fig 14) [29].

Therefore, in situations of increased left atrial pressure, such as in fetuses with diabetes mellitus, twin-totwin transfusion syndrome, and FGR, the parameters of the score should be complemented by others that allow for the detection of cardiac dysfunction. Consequently, it is important to include echocardiographic parameters such as MPI, as well as other alternative evaluation indices of diastolic function that reflect the changes in the atrial dynamics, such as the mobility of the septum primum, PI of the flow of the foramen ovale, AE shortening fraction, PI of the pulmonary vein, PI of VD, and IFI [30].

An LV diastolic dysfunction score was proposed by Zielinsky et al [31] using a model of gestational diabetes mellitus to stratify the risk of perinatal morbidity and mortality of these fetuses. The following formula was proposed to calculate the degree of diastolic dysfunction: score $=$ number of points/total possible points [31]. For example, the use of 4 parameters yields 9 points, which are divided by the total number of points of these 4 parameters and results in percentages that corresponds to the following classification of diastolic dysfunction: absent $(0 \%)$, mild $(1 \%-5 \%)$, moderate $(5 \%-40 \%)$, or severe $(>40 \%)[31]$.

\section{Conclusion}

Fetal functional echocardiography allows for the assessment of cardiac dynamics; the combination of different parameters for assessment of the fetal myocardium may be used to assess cardiac function, including parameters that evaluate the atrial dynamics, and specific applications for certain diseases should always be considered. The analysis of cardiac function provides important information on the hemodynamics and cardiovascular adaptation of the fetus, allows the optimization of the time of delivery, and reduces perinatal morbidity and mortality.

\section{Conflict of interest: none}

\section{References}

1. Volpe P, De Robertis V, Campobasso G, Tempesta A, Volpe G, Rembouskos G. Diagnosis of congenital heart disease by early and second-trimester fetal echocardiography. J Ultrasound Med 2012;31:563-568.

2. Crispi F, Gratácos E. Fetal cardiac function: technical considerations and potential research and clinical applications. Fetal Diagn Ther 2012;32:47-64.

3. Rudolph AM. Circulation in the normal fetus and cardiovascular adaptations to birth. In: Yagel S, Silverman NH, Gembruch U, editors. Fetal Cardiology: Embryology, Genetics, Physiology, Echocardiographic Evaluation, Diagnosis and Perinatal Management of Cardiac Diseases. 2nd ed. Informa Heathcare: New York; 2009:131-151.

4. Kiserud T, Acharya G. The fetal circulation. Prenat Diagn 2004:24:1049-1059.

5. Zielinsky P. The fetus and hermeneutic of the diastole. Arq Bras Cardiol 2002;79:640-643.

6. Huhta JC. Guidelines for the evaluation of heart failure in the fetus with or without hydrops. Pediatr Cardiol 2004;25:274-286.

7. Huhta JC. Fetal congestive heart failure. Sem Fetal Neonatal Med 2005; 10:542-552.

8. Hernandez-Andrade E, Benavides-Serralde JA, Cruz-Martinez R, Welsh A, Mancilla-Ramirez J. Evaluation of conventional Doppler fetal cardiac function parameters: E/A ratios, outflow tracts, and myocardial performance index. Fetal Diagn Ther 2012;32:22-29.

9. Nardozza LM, Rolo LC, Araujo Júnior E, et al. Reference range for fetal interventricular septum area by means of four-dimensional ultrasonography using spatiotemporal image correlation. Fetal Diagn Ther 2013;33:110-115.

10. Passarella G, Trifirò G, Gasparetto M, Moreolo GS, Milanesi O. Disorders in glucidic metabolism and congenital heart diseases: detection and prevention. Pediatr Cardiol 2013;34:931-937.

11. Crispi F, Bijnens B, Figueras F, et al. Fetal growth restriction results in remodeled and less efficient hearts in children. Circulation 2010;121:2427-2436.

12. Van Mieghem T, Hodges R, Jaeggi E, Ryan G. Functional echocardiography in the fetus with non-cardiac disease. Prenat Diagn 2014;34:23-32.

13. Hamill N, Yeo L, Romero R, et al. Fetal cardiac ventricular volume, cardiac output, and ejection fraction determined 
with 4-dimensional ultrasound using spatiotemporal image correlation and virtual organ computer-aided analysis. Am J Obstet Gynecol 2011;205:76.e1-e10.

14. Cruz-Lemini M, Crispi F, Valenzuela-Alcaraz B, et al. Value of annular M-mode displacement vs tissue Doppler velocities to assess cardiac function in intrauterine growth restriction. Ultrasound Obstet Gynecol 2013;42:175-181.

15. Mao YK, Zhao BW, Wang B. Z-Score Reference Ranges for Angular M-Mode Displacement at 22-40 Weeks' Gestation. Fetal Diagn Ther 2016. doi:10.1159/000446071.

16. Messing B, Gilboa Y, Lipschuetz M, Valsky DV, Cohen SM, Yagel S. Fetal tricuspid annular plane systolic excursion (f-TAPSE): evaluation of fetal right heart systolic function with conventional M-mode ultrasound and spatiotemporal image correlation (STIC) M-mode. Ultrasound Obstet Gynecol 2013;42:182-188.

17. Huhta JC, Paul JJ. Doppler in fetal heart failure. Clin Obstet Gynecol 2010;53:915-929.

18. Tei C, Ling LH, Hodge DO, et al. New index of combined systolic and diastolic myocardial performance: a simple and reproducible measure of cardiac function--a study in normals and dilated cardiomyopathy. J Cardiol 1995;26:357-366.

19. Hernandez-Andrade E, Figueroa-Diesel H, Kottman C, et al. Gestational-age-adjusted reference values for the modified myocardial performance index for evaluation of fetal left cardiac function. Ultrasound Obstet Gynecol 2007;29:321-325.

20. Naujorks AA, Zielinsky P, Klein C, et al. Myocardial velocities, dynamics of the septum primum, and placental dysfunction in fetuses with growth restriction. Congenit Heart Dis 2014;9:138-143.

21. Firpo C, Zielinsky P. Behavior of septum primum mobility in third-trimester fetuses with myocardial hypertrophy. Ultrasound Obstet Gynecol 2003;21:445-450.
22. Zielinsky P, Beltrame PA, Manica JL, et al. Dynamics of the septum primum in fetuses with intrauterine growth restriction. J Clin Ultrasound 2009;37:342-346.

23. Zielinsky P, Piccoli A Jr, Gus E, et al. Dynamics of the pulmonary venous flow in the fetus and its association with vascular diameter. Circulation 2003;108:2377-2380.

24. Bravo-Valenzuela NJ, Zielinsky P, Huhta JC, et al. Dynamics of pulmonary venous flow in fetuses with intrauterine growth restriction. Prenat Diagn 2015;35:249-253.

25. Baschat AA, Turan OM, Turan S. Ductus venosus bloodflow patterns: more than meets the eye? Ultrasound Obstet Gynecol 2012;39:598-599.

26. Turan OM, Turan S, Berg C, et al. Duration of persistent abnormal ductus venosus flow and its impact on perinatal outcome in fetal growth restriction. Ultrasound Obstet Gynecol 2011;38:295-302.

27. Fouron JC, Siles A, Montanari L, et al. Feasibility and reliability of Doppler flow recordings in the fetal aortic isthmus: a multicenter evaluation. Ultrasound Obstet Gynecol 2009;33:690-693.

28. Ruskamp J, Fouron JC, Gosselin J, Raboisson MJ, InfanteRivard C, Proulx F. Reference values for an index of fetal aortic isthmus blood flow during the second half of pregnancy. Ultrasound Obstet Gynecol 2003;21:441-444.

29. Hofstaetter C, Hansmann M, Eik-Nes SH, Huhta JC, Luther SL. A cardiovascular profile score in the surveillance of fetal hydrops. J Matern Fetal Neonatal Med 2006;19:407413.

30. Dahlbäck C, Gudmundsson S. Investigations on atrial function in fetuses with signs of impaired placental function. Prenat Diagn 2015;35:605-611.

31. Zielinsky P, Piccoli AL Jr. Myocardial hypertrophy and dysfunction in maternal diabetes. Early Hum Dev 2012;88:273-278. 